POS $\quad$ PROCEEDINGS

\title{
Update on pi and K Physics
}

\section{Bernard*}

Washington University; Saint Louis, Missouri, USA

E-mail: cb@lump.wustl.edu

\section{DeTar, F. Maresca, and J. Osborn}

University of Utah; Salt Lake City, Utah, USA

E-mail: detar@nova.physics.utah.edu, maresca@physics.utah.edu, osborn@physics.utah.edu

\section{Steven Gottlieb and L. Levkova}

Indiana University; Bloomington, Indiana, USA

E-mail: sg@indiana.edu, llevkova@indiana.edu

\section{U.M. Heller}

American Physical Society; Ridge, New York, USA

E-mail: hellerecsit.fsu.edu

\section{J.E. Hetrick}

University of the Pacific; Stockton, California USA

E-mail: jhetrick@uop.edu

\section{Renner and D. Toussaint}

University of Arizona; Tucson, Arizona, USA

E-mail: dru@physics.arizona.edu, doug@klingon.physics.arizona.edu

\section{R. Sugar}

University of California; Santa Barbara, California, USA

E-mail: sugar@physics.ucsb.edu

We present an update of the MILC studies of the physics of light pseudoscalars using improved staggered fermions. New runs at lighter quark mass, as well as increased statistics for older sets, are enabling us to improve the results for decay constants in full QCD. In addition, we have analyzed quenched runs at two different lattice spacings. This makes possible a test of the applicability of staggered chiral perturbation theory in a different context.

XXIIIrd International Symposium on Lattice Field Theory

25-30 July 2005

Trinity College, Dublin, Ireland

\footnotetext{
${ }^{*}$ Speaker.
} 
Since our previously published work [1] on the decay constants and masses of the $\pi-K$ system, we have continued to generate and analyze lattices with $N_{f}=3$ flavors of dynamical quarks, increasing the statistics and moving to lighter $u, d$, and (separately) lighter $s$ masses. We use improved staggered fermions [2]. With the same action, we have also investigated these quantities in the quenched approximation. Assuming the chiral fits are good and the continuum extrapolations are accurate, such calculations make possible:

- A sensitive check of algorithms and methods - including the $\sqrt[4]{\text { Det }}$ trick for dynamical staggered quarks - by comparing full QCD $f_{\pi}$ to the well-determined experimental value.

- A precise extraction of the CKM matrix element $V_{u s}$ from $f_{K}$ or $f_{K} / f_{\pi}$, competitive with the world-average from alternative methods.

- A determination of the light quark masses and their ratios with high lattice precision.

- A test of the applicability of staggered chiral perturbation theory (S $\chi$ PT) $[3,4]$ for describing lattice data - both $N_{f}=3$ and quenched.

- An extraction of the physical coefficients of the $\mathscr{O}\left(p^{4}\right)$ chiral Lagrangian.

- A determination of the extra, unphysical parameters that enter S $\chi$ PT. This is important because these parameters also appear in staggered chiral forms for other physical quantities, e.g., heavy-light decay constants and form factors [5]. Fixing the parameters from the lightlight system reduces the systematic errors in heavy-light computations [6].

In our computations, we analyze two lattice spacings: $a \approx 0.12 \mathrm{fm}$ ("coarse") and $a \approx 0.086 \mathrm{fm}$ ("fine"). We call the valence quark masses are $m_{x}$ and $m_{y}$; the dynamical quark masses (for $N_{f}=3$ ) are $m_{u}^{\prime}=m_{d}^{\prime} \equiv \hat{m}^{\prime}$, and $m_{s}^{\prime}$. Here primes indicate simulation values; the corresponding masses without primes are the physical values. Table 1 shows the lattice parameters used.

The relative lattice scale is determined using the length $r_{1}$ [7] from the static quark potential. We reduce statistical fluctuations in $r_{1} / a$ by fitting to a smooth function. The absolute lattice scale is obtained from the $\Upsilon 2 S$ - $1 S$ splitting. Following the continuum extrapolation in Ref. [8], but using updated HPQCD results [9], rather than those in [10], we obtain $r_{1}=0.318(7) \mathrm{fm}$.

\begin{tabular}{|c|c|c|c|c|c|}
\hline$a m_{q} / a m_{s}$ & $10 / g^{2}$ & size & volume & number & $a(\mathrm{fm})$ \\
\hline $0.03 / 0.05$ & 6.81 & $20^{3} \times 64$ & $(2.4 \mathrm{fm})^{3}$ & 564 & 0.120 \\
$0.02 / 0.05$ & 6.79 & $20^{3} \times 64$ & $(2.4 \mathrm{fm})^{3}$ & 484 & 0.120 \\
$0.01 / 0.05$ & 6.76 & $20^{3} \times 64$ & $(2.4 \mathrm{fm})^{3}$ & 658 & 0.121 \\
$0.007 / 0.05$ & 6.76 & $20^{3} \times 64$ & $(2.4 \mathrm{fm})^{3}$ & 493 & 0.121 \\
$0.005 / 0.05$ & 6.76 & $24^{3} \times 64$ & $(2.9 \mathrm{fm})^{3}$ & $\mathbf{4 0 0}(197)$ & 0.120 \\
\hline $\mathbf{0 . 0 3} / \mathbf{0 . 0 3}$ & $\mathbf{6 . 7 9}$ & $\mathbf{2 0}^{3} \times \mathbf{6 4}$ & $(\mathbf{2 . 4} \mathbf{f m})^{\mathbf{3}}$ & $\mathbf{3 5 0}$ & $\mathbf{0 . 1 2 0}$ \\
$\mathbf{0 . 0 1} / \mathbf{0 . 0 3}$ & $\mathbf{6 . 7 5}$ & $\mathbf{2 0}^{3} \times \mathbf{6 4}$ & $(\mathbf{2 . 4} \mathbf{f m})^{\mathbf{3}}$ & $\mathbf{3 4 9}$ & $\mathbf{0 . 1 2 0}$ \\
\hline quenched & $\mathbf{8 . 0 0}$ & $\mathbf{2 0}^{3} \times \mathbf{6 4}$ & $(\mathbf{2 . 4} \mathbf{f m})^{\mathbf{3}}$ & $\mathbf{4 0 8}$ & $\mathbf{0 . 1 1 9}$ \\
\hline \hline $0.0124 / 0.031$ & 7.11 & $28^{3} \times 96$ & $(2.4 \mathrm{fm})^{3}$ & 531 & 0.0863 \\
$0.0062 / 0.031$ & 7.09 & $28^{3} \times 96$ & $(2.4 \mathrm{fm})^{3}$ & 583 & 0.0861 \\
$\mathbf{0 . 0 0 3 1} / \mathbf{0 . 0 3 1}$ & $\mathbf{7 . 0 8}$ & $\mathbf{4 0}^{3} \times \mathbf{9 6}$ & $(\mathbf{3 . 4} \mathbf{f m})^{\mathbf{3}}$ & $\mathbf{2 1 0}$ & $\mathbf{0 . 0 8 5 9}$ \\
\hline quenched & $\mathbf{8 . 4 0}$ & $\mathbf{2 8} \times \mathbf{3} \times \mathbf{9 6}$ & $(\mathbf{2 . 4} \mathbf{f m})^{3}$ & $\mathbf{3 9 6}$ & $\mathbf{0 . 0 8 5 3}$ \\
\hline
\end{tabular}

Table 1: Lattice parameters. Runs and numbers of configuration in normal font were included in Ref. [1]; those in bold font are new. The lattice sets above the double line are "coarse;" those below are "fine." 
As in Ref. [1] we fit the partially quenched lattice data to S $\chi$ PT forms [4]. The addition of new runs allows us (sometimes forces us) to change some details of the fits. To determine LO and NLO chiral parameters we fit only to the low quark-mass region. The cut on valence quark mass is the same as before: $a m_{x}+a m_{y} \leq 0.021 \approx 0.5 a m_{s}$ (coarse) and $a m_{x}+a m_{y} \leq 0.017 \approx 0.6 a m_{s}$ (fine). We now have enough data to cut on sea-quark masses, too: We remove the $0.03 / 0.05$, $0.02 / 0.05$, and $0.03 / 0.03$ sets for this fit. Because statistical errors are so small, $(0.1 \%$ to $0.4 \%$ for decay constants, $0.1 \%$ to $0.7 \%$ for squared meson masses), we still require NNLO analytic terms in addition to complete NLO forms to get good fits. Such joint fits to decay constants and masses, including both coarse and fine lattices, have 26 free parameters:

- 2 LO parameters: $f$ and $\mu$ (decay constant and condensate at tree-level).

- 8 NLO parameters: 4 physical and 2 taste-violating analytic terms, 2 taste-violating hairpins.

- 10 physical, NNLO analytic parameters.

- 6 tightly constrained parameters (prior width $=0.025$ ): give variation of $2 \mathrm{LO}$ and 4 NLO physical parameters with lattice spacing.

For interpolation around $m_{s}$, we must include higher quark masses. Once the LO and NLO parameters are determined, we fix them (up to statistical errors) and fit to all sea mass sets and wider ranges of valence masses. For central values we choose the range $a m_{x}+a m_{y} \leq 0.055 \approx 1.4 m_{s}$ coarse, and $a m_{x}+a m_{y} \leq 0.0353 \approx 1.3 m_{s}$ fine. This fit is called "Fit C." For systematic error tests, the range is widened to $a m_{x}+a m_{y} \leq 0.10 \approx 2.5 m_{s}$ coarse, and $a m_{x}+a m_{y} \leq 0.062 \approx 2.2 m_{s}$ fine. With either of these choices, we need to add in the NNNLO analytic terms (18 parameters, cubic in quark masses for $f_{\pi}$ and $\left.M_{\pi}^{2} /\left(m_{x}+m_{y}\right)\right)$ to get good fits.

With our old data set, $m_{s}^{\prime}$ only changed with $a$, and $m_{s}^{\prime}$ was usually significantly larger than $\hat{m}^{\prime}$, so the sea quark mass dependence and $a$ dependence were difficult to disentangle. The new data, which includes coarse lattices with $a m_{s}^{\prime}=0.03$ in addition to the previous value $a m_{s}^{\prime}=0.05$, gives better control of the sea quark mass dependence and smaller $a$ dependence of the LO, NLO, and NNLO parameters. Including the NNNLO terms and the $a$ dependence of the NNLO terms gives 28 parameters more than the low-mass fits described above, for a total of 56 parameters. Twelve of these (LO, LO $a$ dependence, and NLO parameters) are tightly constrained from the low-mass fits.

Figure 1 shows Fit $\mathrm{C}$ results for $f_{\pi}$ and $f_{K}$, respectively. This fit has $\chi^{2} /$ d.o.f. $=0.99$ for 556 degrees of freedom (confidence level $\mathrm{CL}=0.59$ ). In each plot, the maroon line is the "prediction" for the 0.0031/.031 fine run based on a second fit that leaves out that run; it should be compared with the solid black line that comes from Fit C. Since the time of the conference, we have accumulated about $25 \%$ more $0.0031 / .031$ configurations, and the effect of removing or including this run in the fit has decreased. Given that the CL decreases when the run is removed, we no longer see any to consider omitting the run. We note that the $0.0031 / .031$ run is still only about half finished, so there will probably be further noticeable shifts. In the $f_{K}$ plot, two "experimental" points (shifted slightly to the left for clarity) are shown. Both points are based on the measured leptonic $(K \rightarrow \ell v)$ rate, but $*$ assumes the PDG value $V_{u s}=0.2200(26)$ [11]; while $\times$ assumes the results of recent experiments $V_{u s}=0.2262(23)[12]$. Both these values of $V_{u s}$ come from experimental determinations of the semileptonic $(K \rightarrow \pi \ell v)$ rate and non-lattice theory for form factors.

Our preliminary results for decay constants are:

$$
f_{\pi}=128.1 \pm 0.5 \pm 2.8 \mathrm{MeV}, \quad f_{K}=153.5 \pm 0.5 \pm 2.9 \mathrm{MeV}, \quad f_{K} / f_{\pi}=1.198(3)\left(\begin{array}{c}
+16 \\
-5
\end{array}\right)
$$



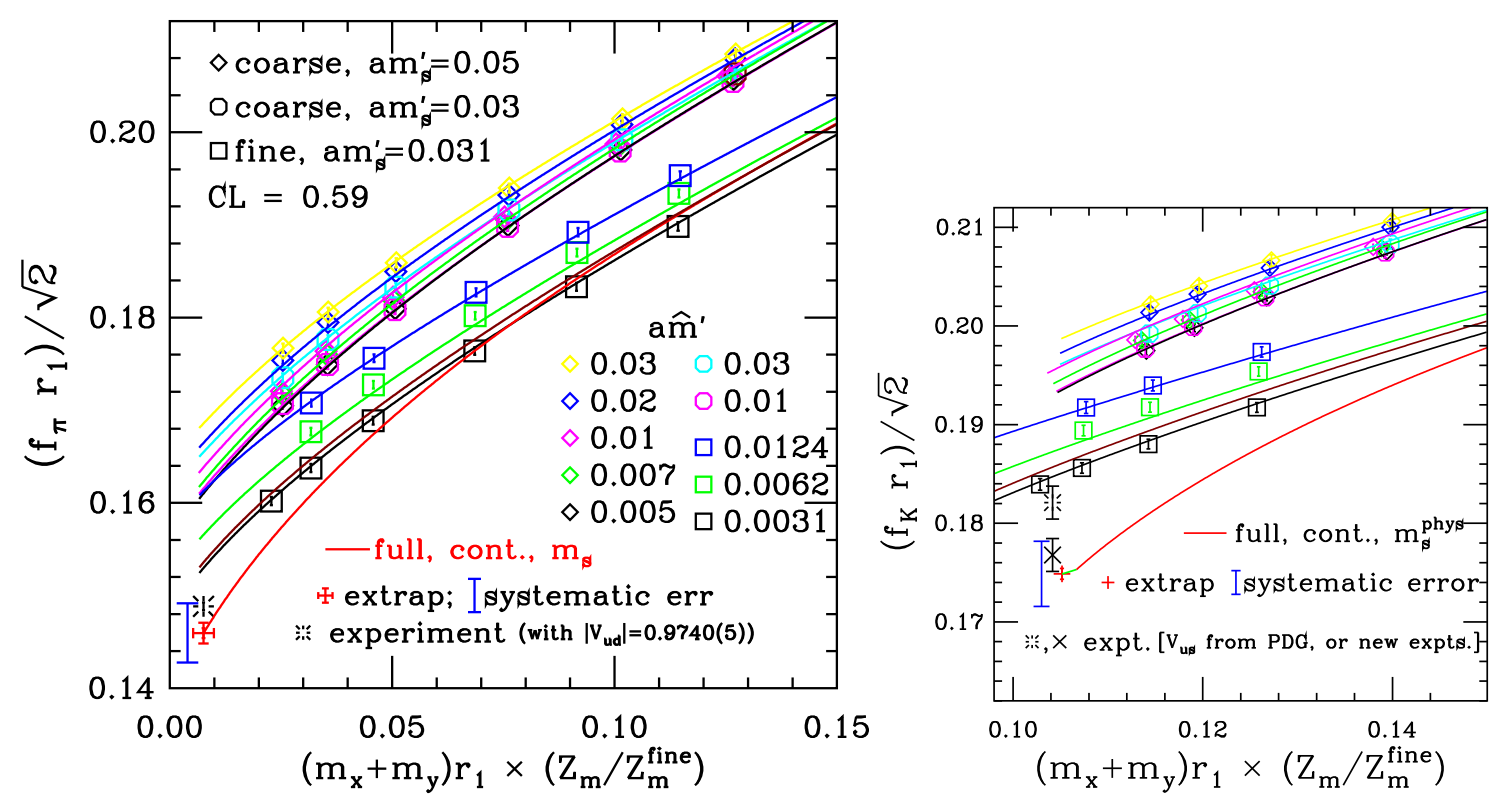

Figure 1: Left: Comparison of Fit $\mathrm{C}$ to partially quenched $f_{\pi}$ data. Extrapolation to continuum, setting $m_{s}^{\prime}=m_{s}$, and going to full QCD gives the red line. Red + shows the final result after extrapolation $m_{x}, m_{y} \rightarrow$ $\hat{m}$. The maroon line (just barely visible above the black line) is the prediction for the $0.0031 / 0.031$ fine run (black squares) based on the other data. Right: Same as left, but for $f_{K}$. The short green continuation of the red line keeps light sea masses fixed at the average physical value $\hat{m}$ and extrapolates $m_{x} \rightarrow m_{u}$.

where the errors are from statistics and lattice systematics. These results are consistent with our previous answers [1], with slightly smaller errors. The current $N_{f}=3$ results for quark masses are little changed from those in Refs. [1, 13].

We extract $V_{u s}$ from our $f_{K} / f_{\pi}$ result. This is probably safer than using $f_{K}$ itself, because the ratio is largely free of scale errors. We obtain $\left|V_{u s}\right|=0.2242\left({ }_{-31}^{+11}\right)$, which is consistent with worldaverage values, with comparably sized errors. From Fig. 1, one can deduce that using $f_{K}$ alone would result in a somewhat higher value of $V_{u s}$. The difference comes from the fact that our $f_{\pi}$ result is slightly low compared with experiment, although consistent within errors. Runs planned for the near future, as well as those now in progress, should allow a further reduction in the errors.

We now turn to the quenched data. We fit to the same valence mass range as the $N_{f}=3$ Fit C. Again, terms through NNNLO terms are needed; joint fits to decay constants and masses, including both coarse and fine lattices, have 34 free parameters:

- 3 LO parameters: $f, \mu$, and the quenched chiral parameter $\delta$ [14]. We consider $\delta$ to be "LO" because its effects are not suppressed by powers of quark mass.

- 7 NLO parameters: 2 physical and 2 taste-violating analytic terms, 2 taste-violating hairpins, and the quenched chiral parameter $\alpha$ [14].

- 4 physical, NNLO analytic parameters.

- 4 physical, NNNLO analytic parameters.

- 14 tightly constrained parameters (prior width $=0.04$ ): give variation of $2 \mathrm{LO}, 4$ NLO, 4 NNLO, and 4 NNNLO physical parameters with lattice spacing.

- 2 parameters to allow the $r_{1}$ scale on the coarse and fine lattices to vary within $1 \sigma$.

The parameter $\delta$ multiplies a function of the taste-singlet mass, which is large ( $\gtrsim 500 \mathrm{MeV}$ ) on coarse lattices because of taste splitting. The coarse lattices are therefore insensitive to quenched 

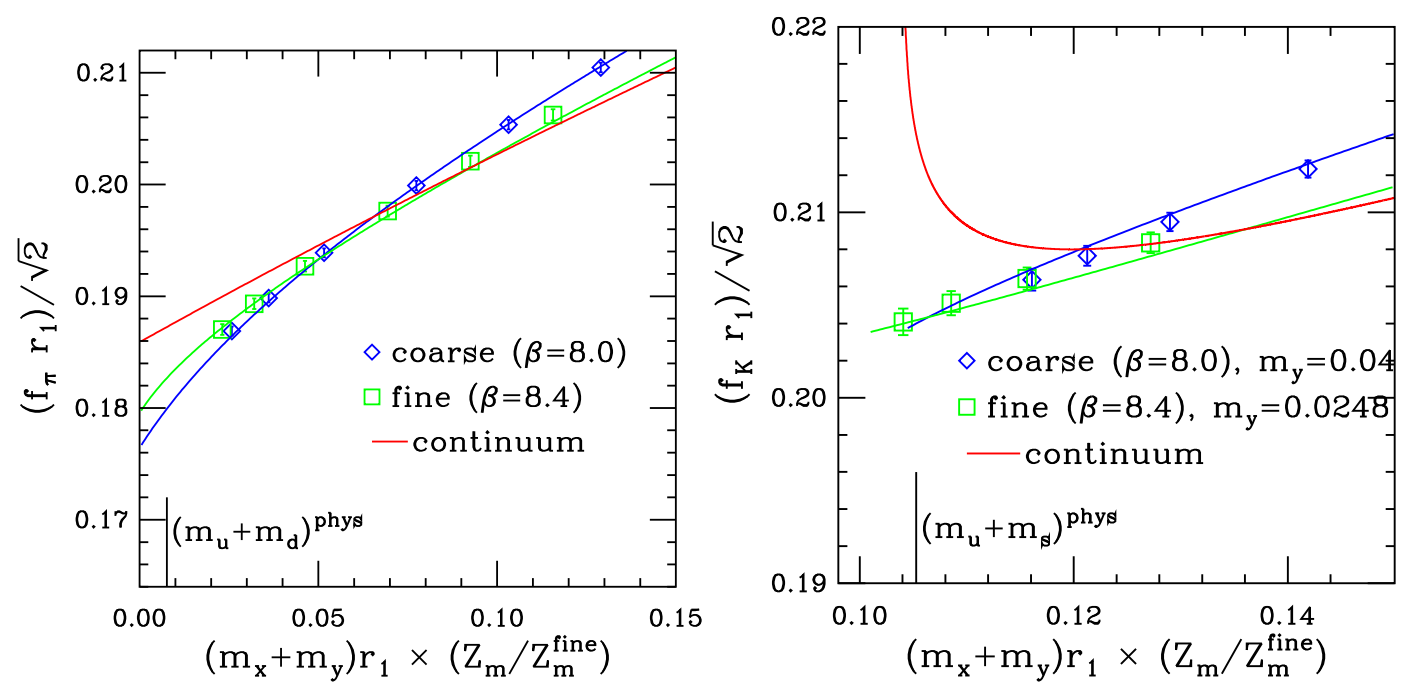

Figure 2: Left: Comparison of Fit $\mathrm{Q}$ to quenched $f_{\pi}$ data. Continuum extrapolation gives the red curve. $\left(m_{u}+m_{d}\right)^{\text {phys }}$ is from the $N_{f}=3$ analysis. Right: Same as left, but for quenched $f_{K}$.

chiral $\operatorname{logs}$, and $\delta$ is poorly determined from coarse-lattice fits: $0.02 \lesssim \delta \lesssim 0.18$, depending on details of the fit and the higher order terms included. Currently, our preferred approach is to obtain $\delta$ from fits to the fine lattices alone, and hold it (as well as the fine lattice scale) fixed in the joint fits. The resulting joint fit, called Fit $\mathrm{Q}$, has 32 parameters and $\chi^{2} /$ d.o.f. $=0.96$ for 98 degrees of freedom; $\mathrm{CL}=0.59$. Comparison of this fit with the quenched $f_{\pi}$ and $f_{K}$ data is shown in Fig. 2.

The continuum-extrapolated curve (red line) blows up at low quark mass for $f_{K}$ but not for $f_{\pi}$ : the latter does not have a 1-loop quenched chiral log in the continuum. Taking into account S $\chi$ PT, $f_{\pi}$ increases significantly with the $a \rightarrow 0$ extrapolation. This makes makes our quenched $f_{\pi}$ differ from experiment (or our $N_{f}=3$ results) by an even larger amount than was reported in Ref. [10] from coarse data alone. In fact, we find $f_{\pi}^{\text {quench }} / f_{\pi}^{N_{f}=3} \approx 1.28$ (with the $r_{1}$ scale). Note, however, that the difference between the raw coarse and fine data for $f_{\pi}$ is small, so it is not yet clear how seriously we should take the continuum extrapolation — data at smaller $a$ is needed. This is an even more important issue for quenched $f_{K}$, where the continuum result blows up in the chiral limit, but the lattice data is smoothed out by S $\chi \mathrm{PT}$ effects. (This is an example of the lack of commutativity of chiral and continuum limits in $\mathrm{S} \chi \mathrm{PT}$ for infrared sensitive quantities [15].)

Values of the taste-violating hairpin parameters in the quenched analysis are comparable to those for $N_{f}=3$. We find:

$$
\begin{array}{ll}
r_{1}^{2} a^{2}\left(\delta_{A}^{\prime}\right)^{\text {quench }}=-0.20(2)\left(_{-8}^{+4}\right) & r_{1}^{2} a^{2}\left(\delta_{A}^{\prime}\right)^{N_{f}=3}=-0.29(1)(4) \\
r_{1}^{2} a^{2}\left(\delta_{V}^{\prime}\right)^{\text {quench }}=0.09(5)\left(_{-7}^{+9}\right) & r_{1}^{2} a^{2}\left(\delta_{V}^{\prime}\right)^{N_{f}=3}=-0.12(2)\left({ }_{-5}^{+11}\right)
\end{array}
$$

In both quenched and $N_{f}=3, \delta_{V}^{\prime}$ is poorly determined and consistent with 0 . For the quenched parameter $\delta$ we are finding: $\delta=0.09(1)(5)$. This result is consistent with most quenched evaluations [16], which get $\delta \approx 0.1$, but not that of the Kentucky group [17], who obtain $\delta=0.24(3)$ (4). (Results in Ref. [18] also tend toward higher values.) Assuming the analysis in Ref. [17], this difference makes sense. Despite the low quark masses in the current calculation, the large tasteviolations in the taste-singlet sector puts us in a larger region of meson mass, where the effective $\delta$ from chiral fits is smaller. Modulo this issue, which needs further study, it seems that S $\chi$ PT works reasonably well in the quenched theory, as it does in the $N_{f}=3$ case. 


\section{References}

[1] C. Aubin et al., Light pseudoscalar decay constants, quark masses, and low energy constants from three-flavor lattice QCD, Phys. Rev. D 70 (2004) 114501 and Results for light pseudoscalars from three-flavor simulations, Nucl. Phys. (Proc. Suppl.) B140 (2005) 231.

[2] K. Orginos, D. Toussaint and R.L. Sugar, Variants of fattening and flavor symmetry restoration, Phys. Rev. D 60 (1999) 054503, and references therein.

[3] W. Lee and S. Sharpe, Partial flavor symmetry restoration for chiral staggered fermions, Phys. Rev. D 60 (1999) 114503; C. Bernard, Chiral logs in the presence of staggered flavor symmetry breaking, Phys. Rev. D 65 (2002) 054031.

[4] C. Aubin and C. Bernard, Pion and kaon masses in staggered chiral perturbation theory, Phys. Rev. D 68 (2003) 034014 and Pseudoscalar decay constants in staggered chiral perturbation theory, Phys. Rev. D 68 (2003) 074011.

[5] C. Aubin and C. Bernard, Staggered chiral perturbation theory with heavy-light mesons, Nucl. Phys. (Proc. Suppl.) B140 (2005) 491, and in preparation.

[6] C. Aubin, et al., Semileptonic decays of D mesons in three-flavor lattice QCD, Phys. Rev. Lett. 94 (2005) 011601 and Charmed meson decay constants in three flavor lattice QCD, Phys. Rev. Lett. 95 (2005) 122002.

[7] R. Sommer, New way to set the energy scale in lattice gauge theories, Nucl. Phys. B411 (1994) 839. C. Bernard et al., The static quark potential in three flavor QCD, Phys. Rev. D 62 (2000) 034503.

[8] C. Aubin et al., Light hadrons with improved staggered quarks: approaching the continuum limit, Phys. Rev. D 70 (2004) 094505.

[9] A. Gray et al., The Upsilon spectrum and m(b) from full lattice QCD, hep-lat/0507013.

[10] C. Davies et al., High precision lattice QCD confronts experiment, Phys. Rev. Lett. 92022001 (2004).

[11] S. Eidelman et al., Review Of Particle Physics, Phys. Lett. B 592 (2004) 1.

[12] E. Blucher, review talk at CKM2005, San Diego, March , 2005.

[13] C. Aubin et al., First determination of the strange and light quark masses from full lattice QCD, Phys. Rev. D 70 (2004) 031504(R).

[14] S. Sharpe, Quenched chiral logarithms, Phys. Rev. D 46 (1992) 3146; C. Bernard and M. Golterman, Chiral perturbation theory for the quenched approximation of QCD, Phys. Rev. D 46 (1992) 853.

[15] C. Bernard, Order of the chiral and continuum limits in staggered chiral perturbation theory, Phys. Rev. D 71 (2005) 094020.

[16] S. Aoki et al., Quenched light hadron spectrum, Phys. Rev. Lett. 84 (2000) 238; W. Bardeen et al., Anomalous chiral behavior in quenched lattice QCD, Phys. Rev. D62 (2000) 114505; M. Göckeler et al., Hadron masses and decay constants in quenched QCD, Nucl. Phys. (Proc. Suppl.) $\mathbf{B 8 3}$ (2000) 203; S. Kim and S. Ohta, Light-hadron spectrum in quenched lattice QCD with staggered quarks, Phys. Rev. D 61 (2000) 074506; Y. Aoki et al., Domain wall fermions with improved gauge actions, Phys. Rev. D 69 (2004) 074504.

[17] Y. Chen et al., Chiral logarithms in quenched QCD, Phys. Rev. D 70 (2004) 034502.

[18] C. Gattringer et al., Quenched spectroscopy with fixed-point and chirally improved fermions, Nucl. Phys. B677 (2004) 3; T.W. Chiu and T.H. Hsieh, Quenched chiral logarithms in lattice QCD with exact chiral symmetry, Phys. Rev. D 66 (2002) 014506. 